Research Article

\title{
Gegen Qinlian Decoction Ameliorates Nonalcoholic Fatty Liver Disease in Rats via Oxidative Stress, Inflammation, and the NLRP3 Signal Axis
}

\author{
Yuqi Ying, ${ }^{1}$ Haitao Zhang, ${ }^{2}$ Dian Yu, ${ }^{1}$ Wei Zhang, ${ }^{3}$ Dongling Zhou $\left(\mathbb{D},{ }^{4}\right.$ \\ and Shuangchun Liu $\mathbb{1}^{5}$ \\ ${ }^{1}$ College of Second Clinical Medicine, Zhejiang Chinese Medical University, Hangzhou 310053, Zhejiang, China \\ ${ }^{2}$ Department of Urinary Surgery, Taizhou Municipal Hospital, Taizhou 318000, Zhejiang, China \\ ${ }^{3}$ Department of Endocrinology, Taizhou Municipal Hospital, Taizhou, Zhejiang, China \\ ${ }^{4}$ Department of Nursing, Taizhou Municipal Hospital, Taizhou, Zhejiang, China \\ ${ }^{5}$ Department of Blood Transfusion Division, Taizhou Municipal Hospital, Taizhou, Zhejiang, China
}

Correspondence should be addressed to Dongling Zhou; zhdl0576@163.com and Shuangchun Liu; 1sc20160412@126.com

Received 15 October 2020; Revised 22 December 2020; Accepted 4 February 2021; Published 16 February 2021

Academic Editor: Vincenzo De Feo

Copyright (c) 2021 Yuqi Ying et al. This is an open access article distributed under the Creative Commons Attribution License, which permits unrestricted use, distribution, and reproduction in any medium, provided the original work is properly cited.

Gegen Qinlian Decoction (GQD), a classic Chinese herbal formula, has been widely used in Chinese clinic for centuries and is well defined in treating nonalcoholic fatty liver disease (NAFLD). However, the mechanism action of GQD on NAFLD is still rarely evaluated. The present study aims to investigate the effect of GQD on treatment of NAFLD in rats and to further explore the underlying mechanism. The rat NAFLD model established by high-fat-diet feeding was used in the research. Our results exhibited the liver lesions and steatosis was significantly alleviated in NAFLD rats treated with GQD via Oil Red O and H\&E staining. Body weight and liver index in GQD groups were reduced significantly $(P<0.05)$. Moreover, the biochemical analyzer test results showed that GQD significantly decreased blood lipid levels total cholesterol (TC), triglycerides (TG), low-density lipoprotein cholesterol (LDL-C), and liver injury indicators alanine aminotransferase (ALT), aspartate aminotransferase (AST), and alkaline phosphatase (ALP), while it increased the level of high-density lipoprotein cholesterol (HDL-C) $(P<0.05)$. The levels of interferon- $\beta$ (IFN- $\beta$ ), tumor necrosis factor- $\alpha$ (TNF- $\alpha$ ), and malondialdehyde (MDA) after the GQD treatment were significantly lower, and then interleukin-2 (IL-2), superoxide dismutase (SOD), and glutathione peroxidase (GSH-Px) levels were lifted significantly $(P<0.05)$. Further, GQD blocked the expression of NLRP3, ASC, caspase-1 mRNA, and proteins in the liver tissues significantly $(P<0.05)$. These findings indicated that GQD can ameliorate the hepatic steatosis and injury of NAFLD. Its possible mechanism involves the modulation of inflammatory cytokines and antioxidative stress and the inhibition of NLRP3 signal axis activation. The results support that GQD may be a promising candidate in the treatment of NAFLD.

\section{Introduction}

Nonalcoholic fatty liver disease (NAFLD) refers to the main clinical manifestation of pathological syndrome caused by excessive liver fat deposition rather than the definite factor drinking $[1,2]$. Its progress period includes simple steatosis, nonalcoholic steatohepatitis (NASH), fibrosis, and cirrhosis $[3,4]$. Nowadays, NAFLD has become one of the common liver diseases, the course of NAFLD varies with the progress, and the main pathological features are steatosis and fat storage [5]. However, there are still not enough safety and effective therapeutic agents so far, and seeking NAFLD potential and promising drugs is always the current focus of attention [6].

Natural herb products are the abundant resources and have been given special attention for NAFLD drug development $[2,7]$. Traditional Chinese Medicine (TCM) has been used clinically since ancient China, and most of TCM compound formulas are composed of herbs. Several TCM formulas or extracts are considered as potential candidates 
in treating NAFLD [2, 7-9]. As is known, the pathological mechanisms of NAFLD are complicated and incompletely understood, and the action of the TCM formulas to antiNAFLD is usually unclear. TCM theories consider most complications of NAFLD as Yang deficiency and phlegmdampness; accordingly, correcting the deficiency and moving the phlegm are effective strategies in treating the disease $[10,11]$. Gegen Qinlian Decoction (GQD), a TCM classical formulation, is derived from the classical legend of TCM, "Treatise on Cold Pathogenic Diseases" (Shanghan Lun in Chinese), written by Zhongjing Zhang in Han Dynasty [12]. It is composed of four TCM herbs: Gegen (Puerariae Lobatae Radix, from Pueraria lobata (Willd.) Ohwi (Fabaceae)), Huangqin (Scutellariae Radix, from Scutellaria baicalensis Georgi (Lamiaceae)), Huanglian (Coptidis Rhizoma from Coptis chinensis (Ranunculaceae)), and Zhigancao (Glycyrrhizae Radix et Rhizoma Praeparata cum Melle from Glycyrrhiza uralensis (Fabaceae)) [13]. GQD has had the effect of clearing away heat and promoting health and relieving muscle and dispersing evil in clinic for thousands of years in East Asia, which matches the strategy described above $[14,15]$. In modern laboratories findings, GQD exerts the function of lowering blood glucose, blood lipid, anti-inflammation, antioxidation, and so forth [16-19]; meanwhile, as reported, GQD can improve nonalcoholic steatohepatitis in rats by resisting insulin and blood lipid or via gut microbiota $[20,21]$. However, the NAFLD therapeutic overall efficacy and molecular mechanism remain to be further evaluated.

It is reported that an important type of receptor NLRP3 inflammatory signal axis exists in innate immunity, named, NLRP3-ASC-caspase-1 signal axis (NLRP3 signal axis) $[22,23]$, which can mediate NAFLD-induced inflammation and oxidative stress response $[24,25]$. In the present study, we used high-fat-induced NAFLD rats to evaluate the effect of TCM intervention. The paper intends to determine the levels of serum lipids, inflammatory factors, and antioxidant factors of Gegen Qinlian Decoction (GQD) in NAFLD rats and further investigate its regulatory effect on NLRP3 signal axis to explore the potential mechanism of GQD intervention in rat NAFLD.

\section{Materials and Methods}

2.1. Chemicals and Reagents. The four Chinese herbs of GQD were purchased from Chinese Herbal Medicine Co., Ltd. of Zhejiang Chinese Medicine University (Lot: 20181213, 20160523, 20160718, and 20181025, respectively) and certified by Professor Zhang Shuili, an Associate Professor at the School of Pharmacy, Zhejiang Chinese Medicine University (Hangzhou, China), in addition to atorvastatin (Lot: PZ0001, Sigma-Aldrich, USA), rat triglyceride (TG), low-density lipoprotein cholesterol (LDL-C) kit (Lot: 20180324 and 20180330, Shanghai Fusheng Industrial Co., Ltd. China), TRIzol Reagent (Lot: 20150502, Invitrogen Co., USA), SYBR Green PCR and Reverse Transcription kit (Lot: A5001-1, AA406-1; TaKaRa Co., Japan), rabbit anti-rat primary antibodies NLRP3, ASC, and caspase-1 (Lot: 08063965, 2813878, and GR202191-1; Abcam
Co., UK), rabbit anti-rat GAPDH primary antibody, horseradish peroxidase- (HRP-) labeled goat anti-rabbit secondary antibody IgG, and protein molecular mass marker and BCA protein quantification kit (Lot: 00021408, 00081307, 00131456, and 23072018; Kangwei Century Biotechnology Co., Ltd., Beijing, China).

2.2. Preparation of GQD. GQD extracts were prepared according to the method of the Chinese Pharmacopoeia [13]. The herbal material Pueraria Lobatae Radix $(250 \mathrm{~g})$ was soaked in $2 \mathrm{~L}$ of cold water for $30 \mathrm{~min}$ before being boiled for $30 \mathrm{~min}$ alone. The other herbs, including Scutellariae Radix (150 g), Coptidis Rhizoma (150 g), and Glycyrrhiza Radix $(100 \mathrm{~g})$, were added and boiled together with Pueraria Lobatae Radix for $60 \mathrm{~min}$. The first decoction was thus obtained. The mixture was boiled a second time with an additional $1.5 \mathrm{~L}$ of water for $30 \mathrm{~min}$ to obtain the second decoction. Finally, the first and second decoctions were mixed, filtered through gauze, and concentrated to a final extract in a weight ratio of $5: 3: 3: 2$ [20]. Extensive chemical studies have mentioned that flavones (free form and glycosides), flavanones, alkaloids, and triterpene saponins are the major compounds in GQD, especially the active ingredients, baicalin, and berberine hydrochloride [26].

2.3. Animal Model and Experimental Design. Specific Pathogen-Free (SPF) male Sprague Dawley (SD) rats, weighing $180 \pm 20 \mathrm{~g}$, were supplied from the Animal Experimental Center, Zhejiang Academy of Medical Sciences, China [Certificate no. SCXK (Zhe) 2018-0033]. The rats were housed in a circumstance with controlled temperature $\left(20 \sim 25^{\circ} \mathrm{C}\right)$ and humidity (40 45\%) and a $12: 12 \mathrm{~h}$ light/dark cycle. Rats were supplied with granular food and could access water freely as normal rodents. The experiments were conducted in accordance with local guidelines for experimental animal care in Zhejiang Chinese Medicine University, which were approved by the Institutional Research Ethical Committee for the use of laboratory animals.

After SD rats were fed adaptively in the environment of $12 \mathrm{~h}$ and $26^{\circ} \mathrm{C}$ for 7 days, they were randomly divided into a control group, a model group, an atorvastatin group, and GQD high-, medium-, and low-dose group, with 10 rats in each group. According to the previous reports [20, 27, 28] and preliminary experiments, the high, medium, and low doses of GQD were determined to be 8,4 , and $2 \mathrm{~g} / \mathrm{kg} / \mathrm{d}$ (about 1,2, and 4 times of human dose, respectively), and atorvastatin was $2.0 \mathrm{mg} / \mathrm{kg} / \mathrm{d}$. Except that the control group uses common feed, the model group and each treatment group are fed with high-fat diet (ratio of fat:protein: carbohydrate is $3: 1: 1)$ for nonalcoholic fatty liver modeling. After 8 weeks, gavage treatment was performed according to the above dose, with a gavage volume of $2.0 \mathrm{~mL} /$ $100 \mathrm{~g}$; the control group and the model group were given $0.9 \%$ saline in equal volume and gavage once a day for 4 weeks. Animals were weighed every week. They were fasted for $12 \mathrm{~h}$ before the last dose and anesthetized with pentobarbital sodium, blood was taken from the abdominal aorta, 
and serum was centrifuged; the rats were sacrificed and dissected to collect the liver.

\subsection{Histopathological Section Observation by Oil Red O} Staining. The liver tissues of rats were collected and washed in normal saline at $4^{\circ} \mathrm{C}$, and the surface water was blotted dry with filter paper and then immediately put into $4 \%$ formalin solution for fixation. After 1 week, the specimens were embedded in paraffin and regular sections (thickness $4 \mu \mathrm{m}$ ); then the slices were placed in the Oil Red $\mathrm{O}$ and hematoxylin \& eosin (H\&E) staining solution and rinsed with distilled water for $30 \mathrm{~min}$, and finally the slides were sealed. All the samples were examined and observed under an optical microscope.

2.5. Serum Biochemical Analysis. A Hitachi automatic biochemical analyzer (Wako, Richmond, VA, USA) was used to detect serum lipid levels in rats total cholesterol (TC), triglycerides (TG), low-density lipoprotein cholesterol (LDL$\mathrm{C})$, and high-density lipoprotein cholesterol (HDL-C), as well as liver injury indicators alanine aminotransferase (ALT), aspartate aminotransferase (AST), and alkaline phosphatase (ALP). Meanwhile, ELISA assay was used for serum inflammatory cytokines interleukin-2 (IL-2), interferon- $\beta$ (IFN- $\beta$ ), tumor necrosis factor- $\alpha$ (TNF- $\alpha$ ), and antioxidant makers superoxide dismutase (SOD), glutathione peroxidase (GSH-Px), and malondialdehyde (MDA) expression (Lot: 20170655IL2M, 20175200IFNM, 20170052TNFM, 20176020NOM, 20174899SODM, and 20146660GSHM, respectively; R\&D Co., USA).

2.6. Real-Time Quantitative PCR Assay to Test the Key Target Genes of NLRP3 Signaling Axis in Liver Tissue. The tissues around the rat liver were removed and homogenized quickly. The total RNA was extracted according to the instructions of the RNA extraction kit; then the total RNA concentration and absorbance A were measured. Each RNA sample was reversely transcribed as cDNA through the reverse transcription kit. The above cDNA was subjected to PCR amplification under the following conditions: predenaturation at $95^{\circ} \mathrm{C}$ for $10 \mathrm{~min}$, denaturation at $95^{\circ} \mathrm{C}$ for $15 \mathrm{~s}$, annealing at $60^{\circ} \mathrm{C}$, and extension for $45 \mathrm{~s}$, for a total of 40 cycles. GAPDH was used as an internal reference gene to detect NLRP3, ASC, and caspase-1 gene expression on a real-time PCR detector. The Ct value of each sample is obtained by instrument analysis, and all sample genes are normalized by the internal reference GAPDH, and the $2^{-\triangle \triangle \mathrm{Ct}}$ method is used to calculate mRNA relative expression. $\triangle \triangle \mathrm{Ct}$ is given in [29]. The primers were synthesized by Shanghai Bioengineering Co., Ltd., and the primer sequences are shown in Table 1.

2.7. Western Blotting Assay to Determine the NLRP3 Signaling Axis Key Target Proteins in Liver Tissue. Liver tissues were quickly homogenized; then RIPA lysate was added at a ratio of $1: 10(\mathrm{~g} / \mathrm{ml})$, and the total protein content was measured according to the instructions of the BCA protein quantification kit, to ensure that the sample volume is the same. After the protein of each group was lysated and extracted, it was separated by SDS-PAGE electrophoresis and transferred to nitrocellulose membrane, and the converted PVDF membrane was blocked in 5\% skim milk powder solution at room temperature for $2 \mathrm{~h}$, and TBST washing solution was rinsed for $3 \sim 4$ times. Then the following procedure was launched: Add primary antibody and incubate overnight at $4^{\circ} \mathrm{C}$. Rinse 3 to 4 times, add HRPlabeled secondary antibody, incubate at room temperature for $2 \mathrm{~h}$, and rinse 3 to 4 times. Chemiluminescence detection substrate ECL working solution was used for color development. ImageJ analysis system was used to analyze the protein western blot in grayscale, and the protein expression levels of NLRP3, ASC, and caspase-1 in the liver tissue from each group were tested and compared.

2.8. Statistical Analysis. The experimental data were processed by SPSS 17.0 software. ELISA data analysis was carried out using ELISA Calc regression/fitting computing software, and the results were expressed as the mean\pm standard deviation ( $\mathrm{x} \pm \mathrm{s}$ ). The two-tailed Student's $t$-test was used to compare two groups and one-way ANOVA with Tukey's post hoc test was performed for the comparison among multiple groups. $P<0.05$ indicated that the difference was significant.

\section{Results}

3.1. Effects of $G Q D$ on NAFLD in Rats. To evaluate the effects of GQD on treatment of NAFLD rats, the body weight and liver index were monitored. As shown in $\mathrm{A}$ and $\mathrm{B}$ in Figure 1, the body weight and liver index of NAFLD rats after GQD intervention showed a significant mitigation compared with model group $(P<0.05)$. In addition, this effect among the high, medium, and low doses of GQD group reflected a dose-effect relationship. Thereby, the low dose of GQD group seemed to exhibit the intervention but not obviously.

The Oil Red $\mathrm{O}$ and H\&E staining results showed that the liver slices of the control group showed no obvious lesions and no lipid droplets. Compared with the control group, the model group had significant obvious increases in steatosis and lipid droplets, with noticeable hepatic steatosis and infiltration of inflammatory cells in the centrilobular and portal areas, which also confirmed the success in establishing the nonalcoholic fatty liver model. The GQD groups had improved lipid droplets and lesions in different degrees compared with the model group. From pathological slices, it could be seen that the GQD high-dose group showed the best improvement, close to atorvastatin. It shows that GQD can improve the lipid storage of rat liver caused by high-fat diet and reduce hepatic steatosis. The results are shown in Figures 1(c) and 1(d).

3.2. Effects of GQD on Serum Biochemical Parameters in NAFLD Rats. GQD showed a favorable therapeutic efficacy in NAFLD; in order to investigate the regulation of NAFLD- 
TABLE 1: Real-time fluorescence quantitative PCR primer sequence.

\begin{tabular}{|c|c|c|c|}
\hline Gene & & mer sequences & Primer length \\
\hline NLRP3 & $\begin{array}{l}\text { Forward } \\
\text { Reverse }\end{array}$ & $\begin{array}{l}\text { 5' CTCGCATTGGTTCTGAGCTCA 3' } \\
\text { 5’ AGTAAGGCCGGAATTCACCA 3' }\end{array}$ & $153 \mathrm{bp}$ \\
\hline ASC & $\begin{array}{l}\text { Forward } \\
\text { Reverse }\end{array}$ & $\begin{array}{l}\text { 5' TGGAGTCGTATGGCTTGGAG3' } \\
\text { 5' TGTCCTTCAGTCAGCACACT3' }\end{array}$ & $300 \mathrm{bp}$ \\
\hline Caspase-1 & $\begin{array}{l}\text { Forward } \\
\text { Reverse }\end{array}$ & $\begin{array}{l}\text { 5' ACTCGTACACGTCTTGCCCTC 3' } \\
\text { 5' CTGGGCAGGCAGCAAATTC 3' }\end{array}$ & $190 \mathrm{bp}$ \\
\hline GAPDH & $\begin{array}{l}\text { Forward } \\
\text { Reverse }\end{array}$ & $\begin{array}{l}\text { 5' GTGACACCCACTCTTCCACC 3' } \\
\text { 5' GTGGTCCAGGAGGCTCTTAC 3' }\end{array}$ & $162 \mathrm{bp}$ \\
\hline
\end{tabular}

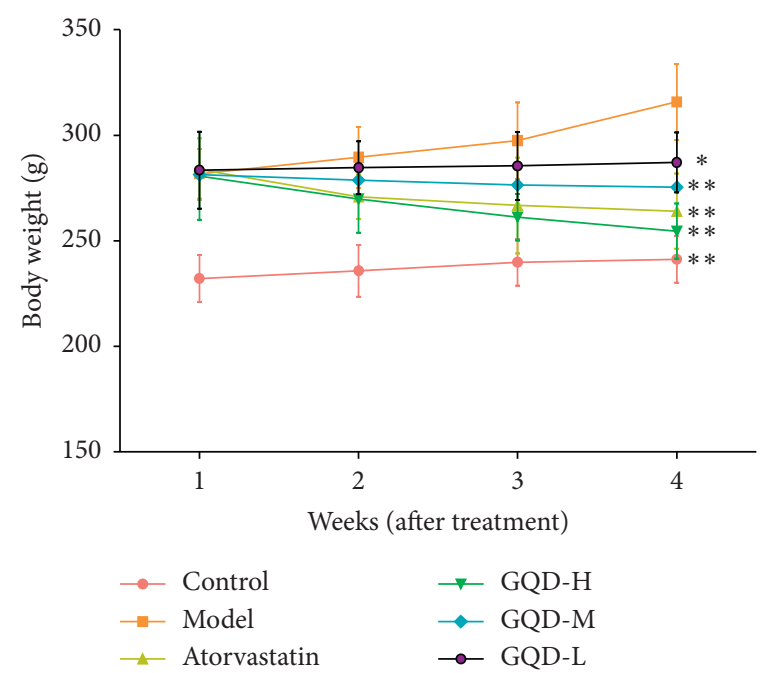

(a)

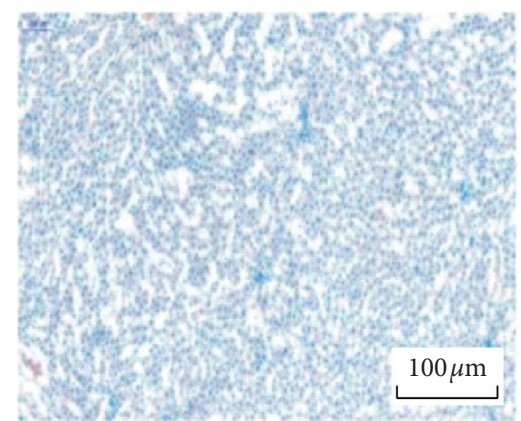

Control

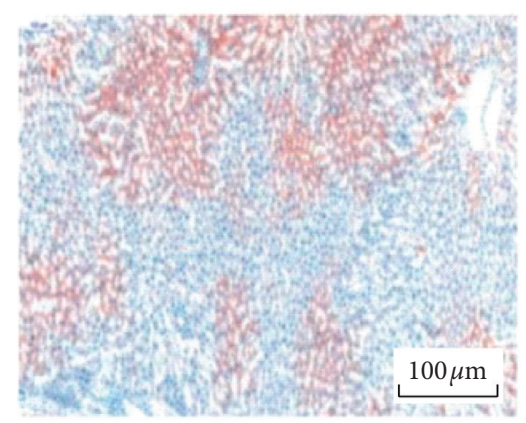

GQD-H

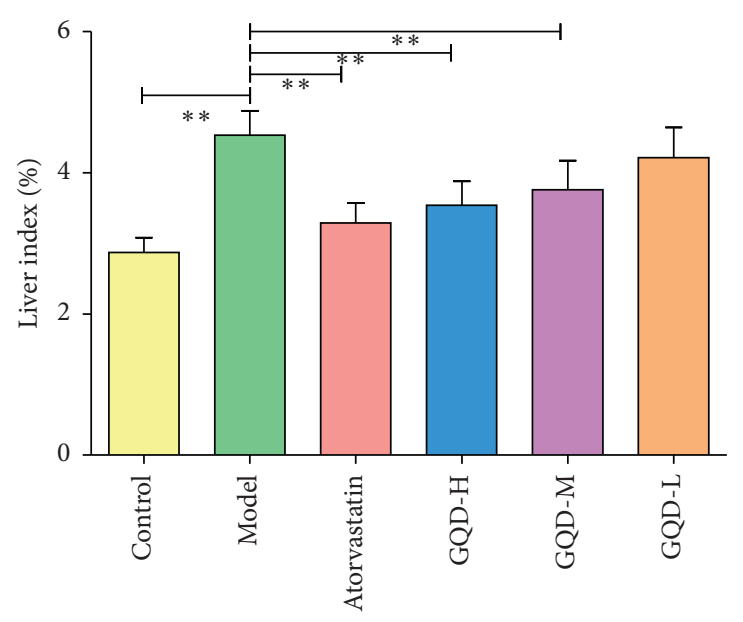

(b)

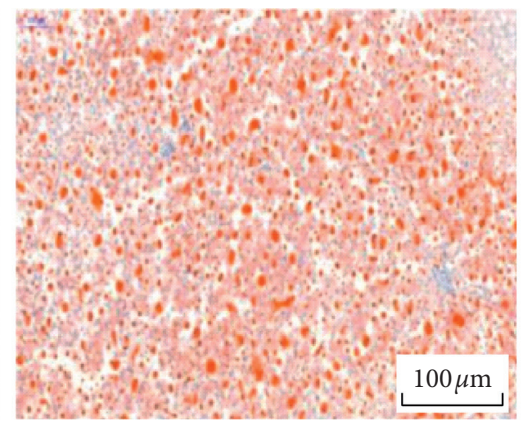

Model

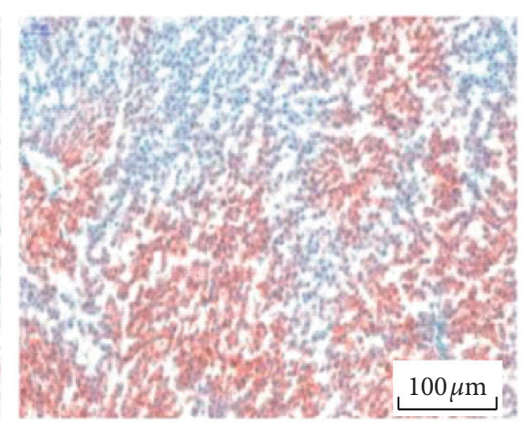

GQD-M

(c)

Figure 1: Continued. 


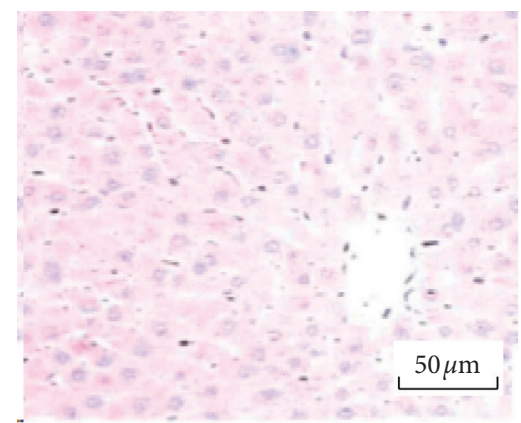

Control

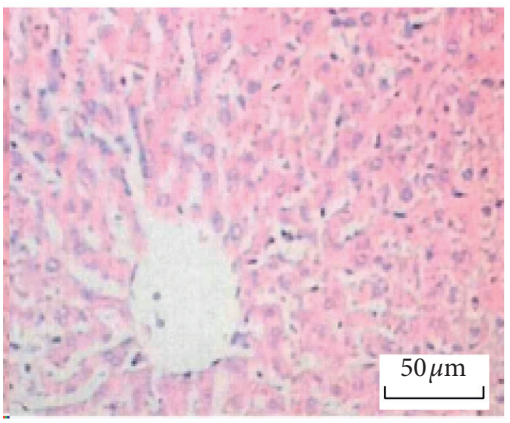

GQD-H

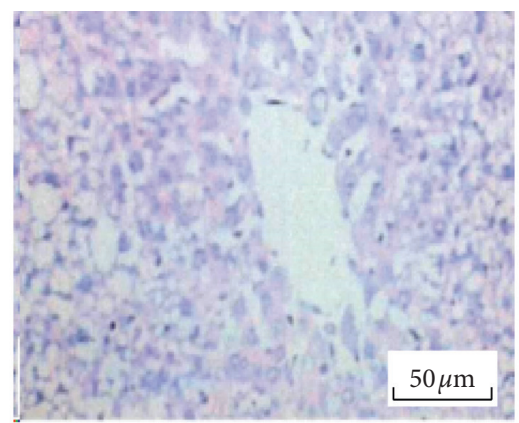

Model

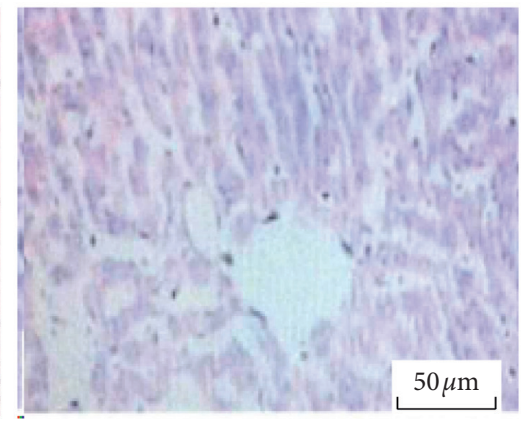

GQD-M

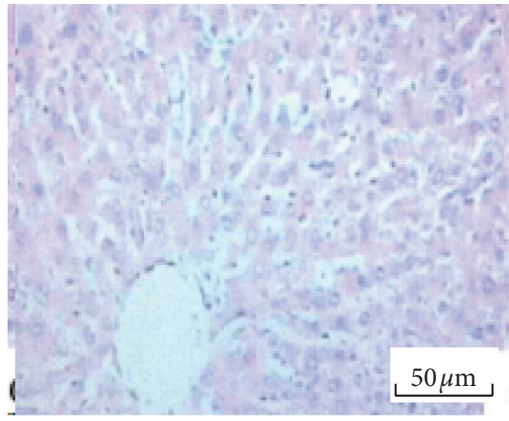

Atorvastatin

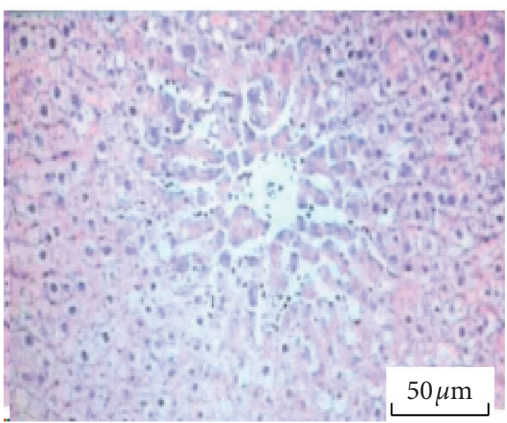

GQD-L

(d)

FIGURE 1: GQD alleviated the NAFLD in rats. (a) Effect on body weight. (b) Effect on liver index. (c) Histological section observation of liver tissues by Oil Red O staining (magnification x200). (d) Histopathological examination of liver tissues by H\&E staining (magnification $\times 400$ ).

related biomolecules in rats, the serum TG, LDL-C, TC, HDL-C, ALT, AST, and ALP were detected. The results showed that the blood lipid expressions of TG, TG, and LDL-C were increased in the model group, while HDL-C was significantly decreased. The liver injury indicators levels ALT, AST, and ALP in the model group were significantly increased compared with the control group, respectively $\left(P^{<} 0.05\right)$. The GQD high-, medium-, low-dose group in blood lipid levels, compared with the model group, had significantly decreased TG, TG, and LDL-C levels, while HDL-C was increased $(P<0.05)$. Moreover, the liver injury indicators levels ALT, AST, and ALP in GQD groups were decreased significantly $(P<0.05)$. These showed that GQD can modulate the metabolism of blood lipid and liver injury indicators to ameliorate NAFLD in rats (Figure 2).

\subsection{GQD Mediated Inflammatory Cytokines and Antioxidant} Markers. To explore the modulation in inflammation and oxidative stress of the GQD on NAFLD, cytokines and antioxidant markers levels test was performed (Figure 3). Compared with the control group, the levels of IFN- $\beta$, TNF$\alpha$, and MDA in the model group were significantly increased, while the levels of IL-2, SOD, and GSH-Px were significantly reduced $(P<0.01)$. The GQD groups lowered the expression of IFN- $\beta$, TNF- $\alpha$, and MDA in rats to different degrees and the expressions of IL-2, SOD, and GSH-Px were increased $(P<0.05)$; and the high-dose group had the strongest regulation effect. It indicated that GQD could suppress the expression levels of IFN- $\beta$, TNF- $\alpha$, and MDA in rat serum and increase the expression levels of IL-2, SOD, and GSH$\mathrm{Px}$, suggesting that GQD can modulate the inflammation and block the oxidative stress in NAFLD rats.

3.4. GQD Inhibited the NLRP3 Signaling Axis of Liver Tissue in NAFLD Rats. GQD exposure for 4 weeks significantly suppressed the inflammation and oxidative stress, which could be mediated by NLRP3 inflammasome as previously reported [24]. Meanwhile the involvement of NLRP3 inflammasome related signal axis activation in NAFLD progression was clearly shown $[24,25]$. In comparison with control, the expression levels of NLRP3, ASC, caspase-1 protein, and mRNA in liver tissue from the model group were significantly increased $(P<0.01)$; compared with the model group, the expression levels of NLRP3, ASC, caspase1 protein, and mRNA were significantly reduced (except the expression of ASC protein in the low-dose group of GQD, all $P<0.05)$; the expression level of each GQD group was higher than that of atorvastatin group (shown in Figure 4 and Supplementary Figure S1). It showed that GQD can inhibit the activation of NLRP3/ASC/caspase-1 signal axis.

\section{Discussion}

The pathogenesis of nonalcoholic fatty liver disease (NAFLD) is complex, involved with hepatic parenchymal cells, nonparenchymal cells, immune cells, and multiple cytokines or pathways, and often further develops into more serious diseases such as cirrhosis and liver cancer [30, 31]. 


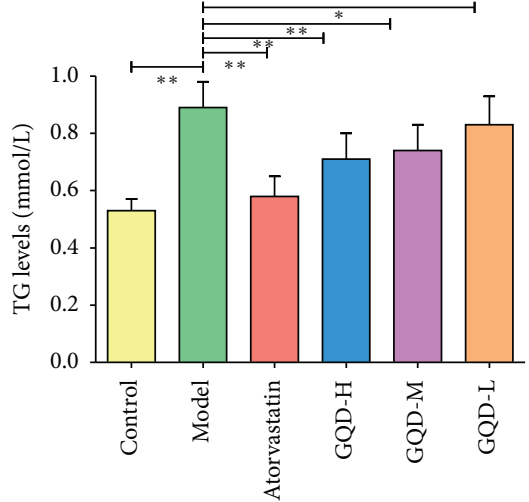

(a)

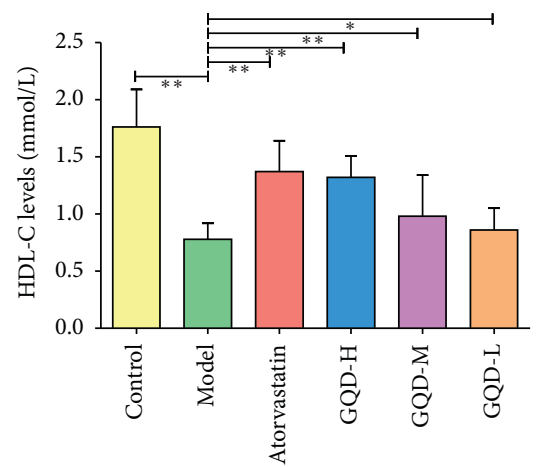

(d)

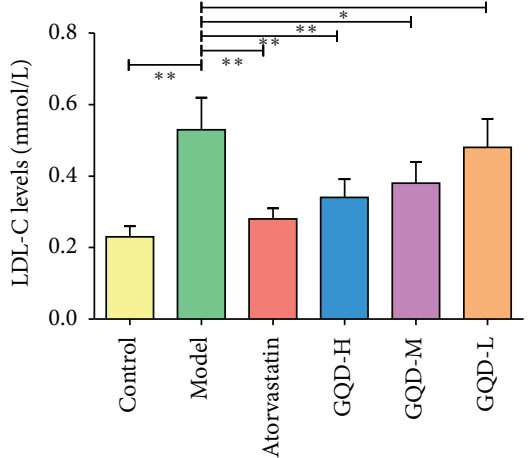

(b)

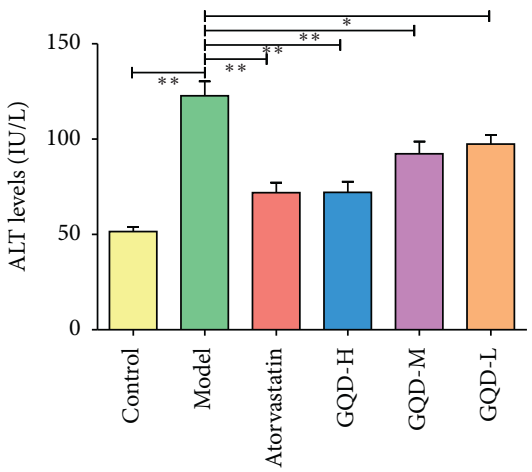

(e)

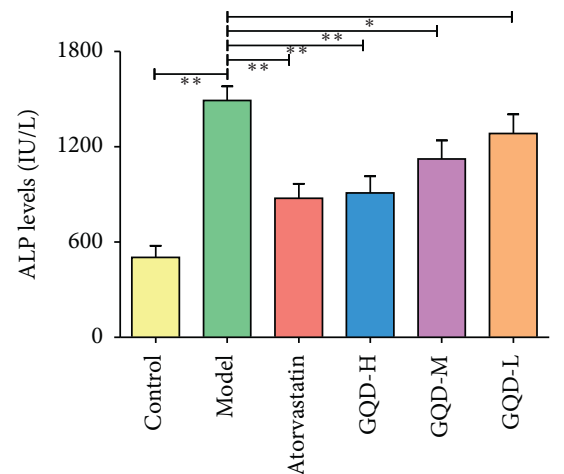

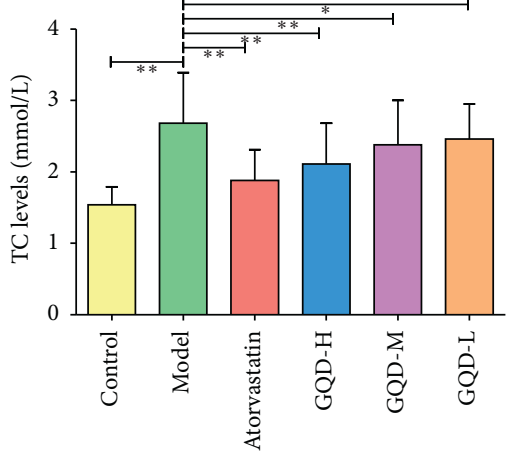

(c)

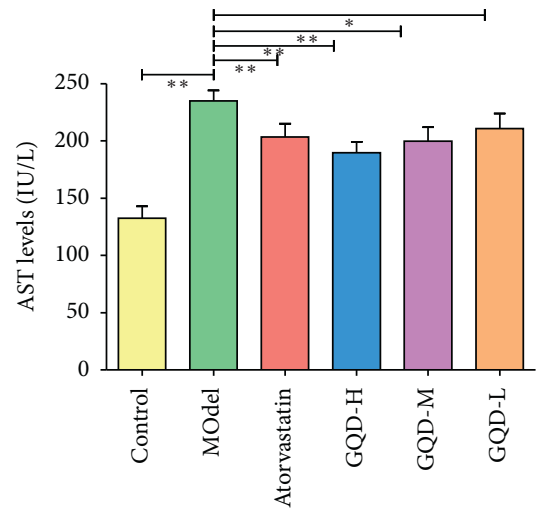

(f)

(g)

Figure 2: The effect of GQD on blood lipid related indexes in NAFLD rats $(n=10)((\mathrm{a})$ TG, (b) LDL-C, (c) TC, (d) HDL-C, (e) ALT, (f) AST, and (g) ALP). Note: compared with the model group, ${ }^{*} P<0.05 ;{ }^{* *} P<0.01$.

Those key targets of pathophysiological mechanisms are important for the development of noninvasive markers of NASH and liver fibrosis as well as targeted therapeutic drugs. The present clinical treatment mainly aims at single target and pathway, and the curative effect is not satisfactory. Therefore, the development of safe and effective drugs, especially drugs combined with multiple factors and links in the progression of NASH and liver fibrosis, has become a new strategy for the treatment of NAFLD $[32,33]$. Traditional Chinese Medicine (TCM) is known for its characteristics of multiple pathways and multiple targets, and TCM has relatively few side effects in the treatment of NAFLD [34]. As reported, GQD alleviates nonalcoholic steatohepatitis associated liver injuries via anti-inflammatory response and inhibition of toll-like receptor 4 signaling pathways [20]. In the present study, the TCM prescription Gegen Qinlian Decoction (GQD) was taken to study its intervention effect on NAFLD in rats. Regarding this, it is generally believed that the progression of hepatic fibrosis can be used as one of the most important endpoints to judge the efficacy of drugs [32]. The pathological analysis results showed that GQD could significantly relieve liver disease and lipid degeneration in rats and block the NAFLD progression. The results of histopathology indicated that GQD exhibited a promising mitigation on fatty disorders of NAFLD. 

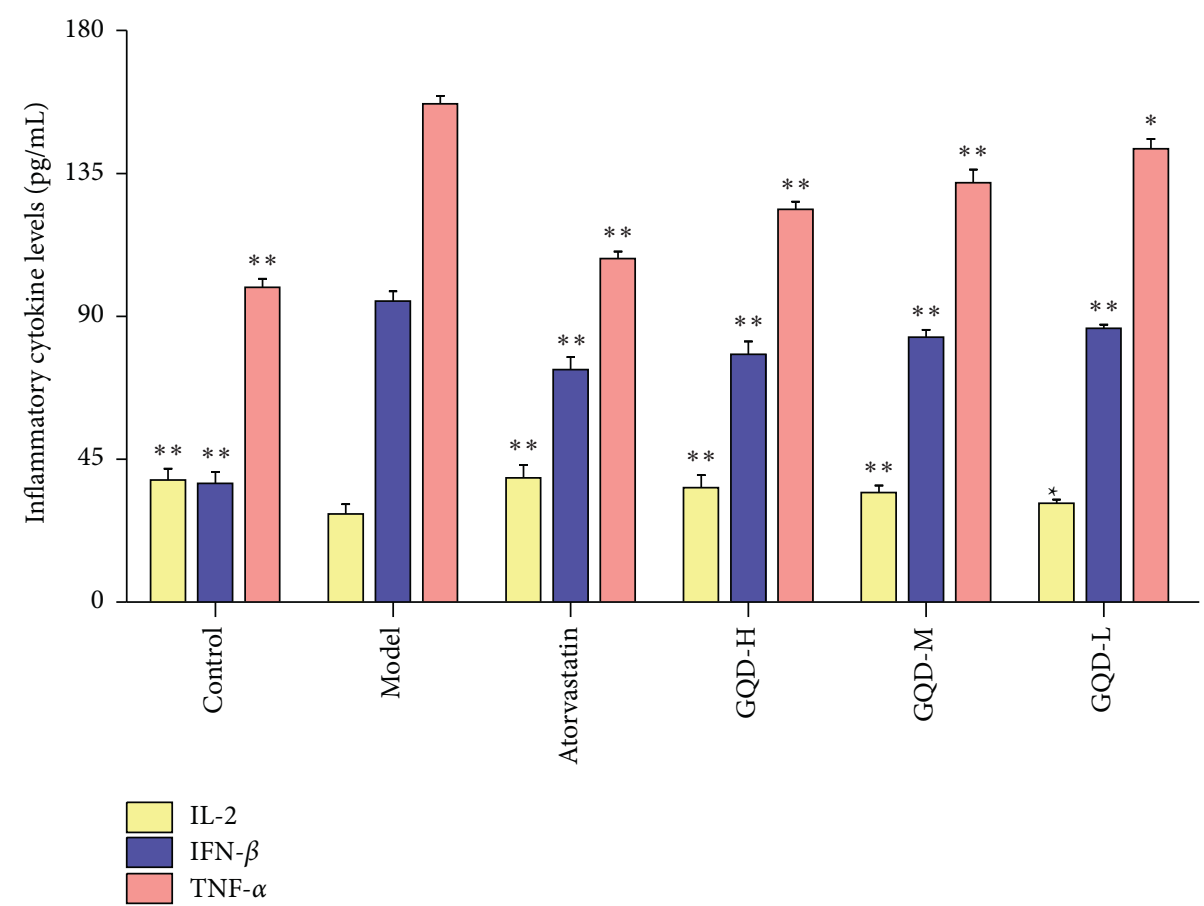

(a)

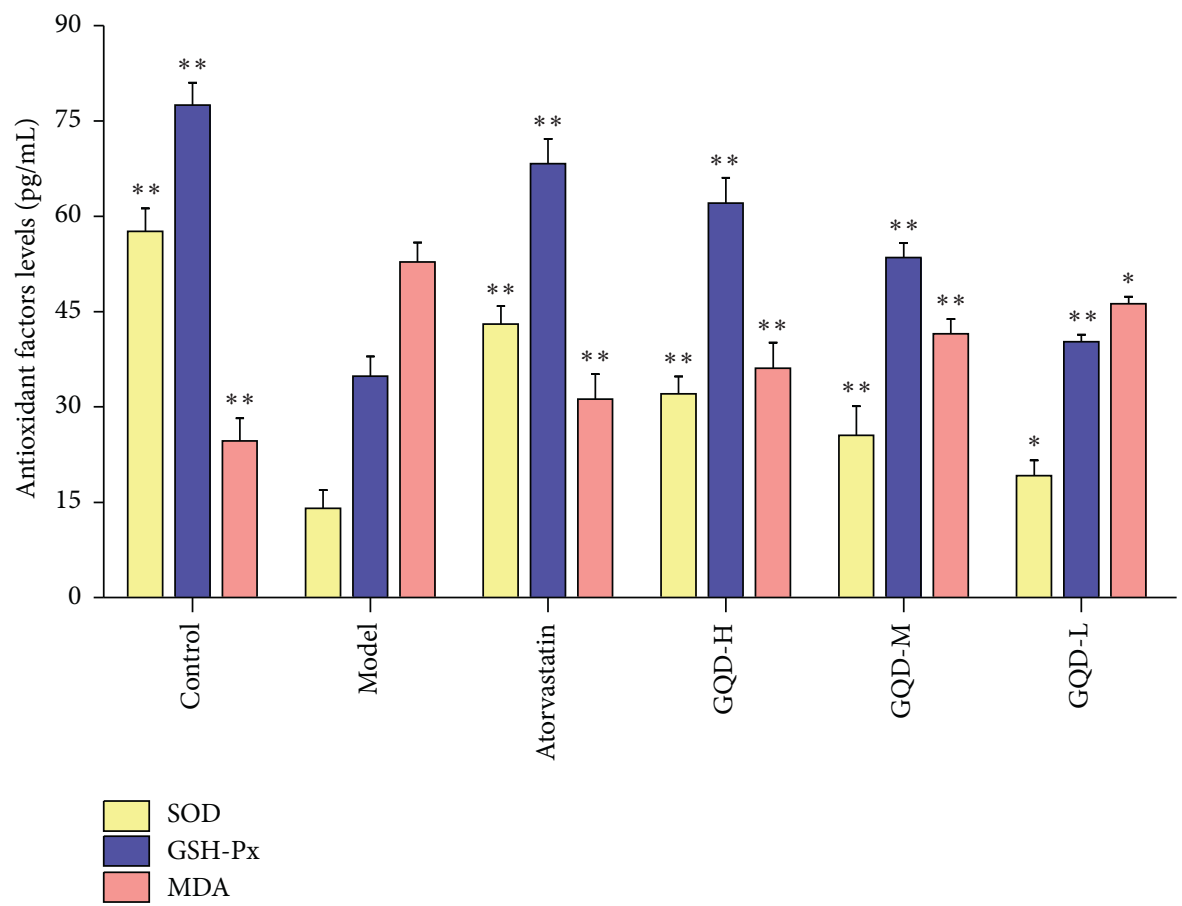

(b)

FIGURE 3: Effects of GQD on serum inflammatory cytokines and antioxidant factors in NAFLD rats $(n=10)$. (a) Regulation of GQD on rat serum IL-2, IFN- $\beta$, and TNF- $\alpha$ levels. (b) Effects of GQD on SOD, GSH-Px, and MDA secretion. Note: compared with the model group, ${ }^{*} P<0.05:{ }^{* *} P<0.01$.

The prestage of NAFLD is mainly the accumulation of lipid droplets, mainly due to the excessive accumulation of TC, TG, LDL-C, and other lipids in serum and/or liver, and the decrease of HDL-C, which usually occurs when a large number of lipids influx and exceed liver lipid clearance
$[35,36]$. Moreover, AST, ALT, and ALP are common indicators of liver metabolism and transport function, and once liver function is impaired, its serum level can be significantly increased $[37,38]$. The World Health Organization (WHO) considers ALT as the most sensitive indicator of 


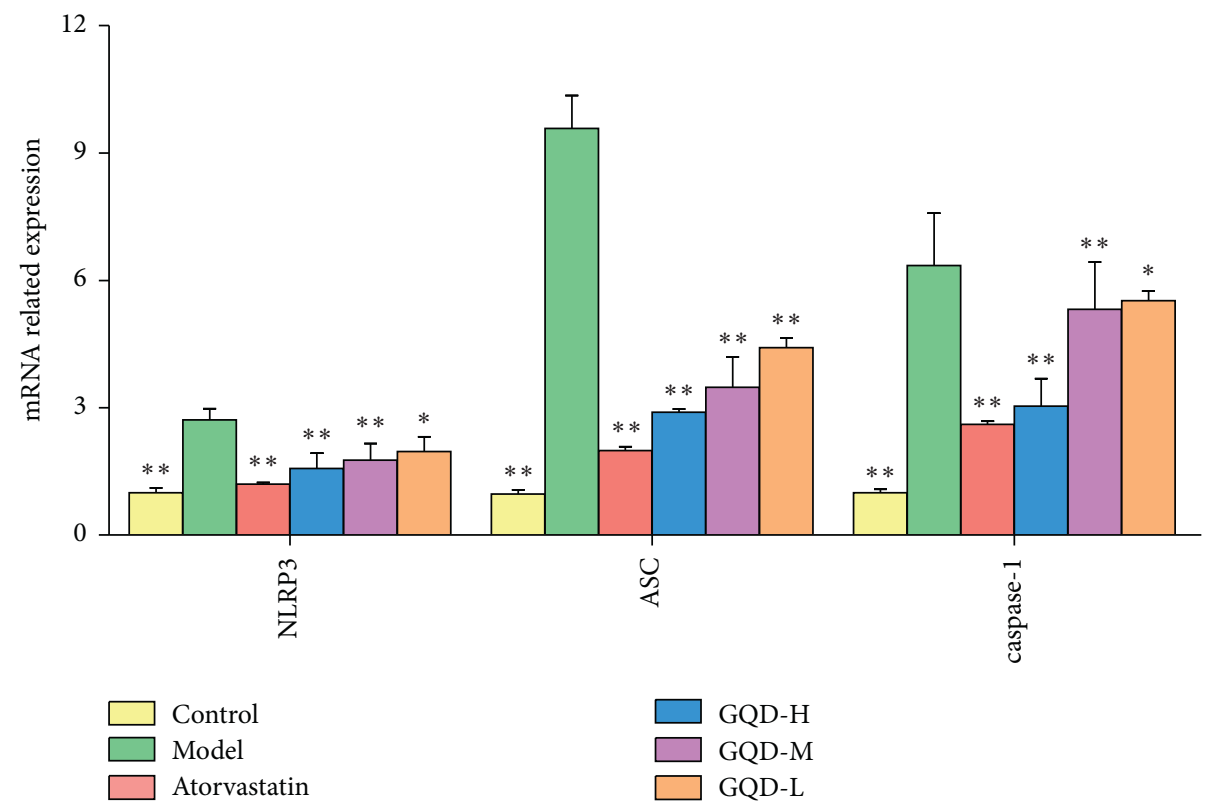

(a)

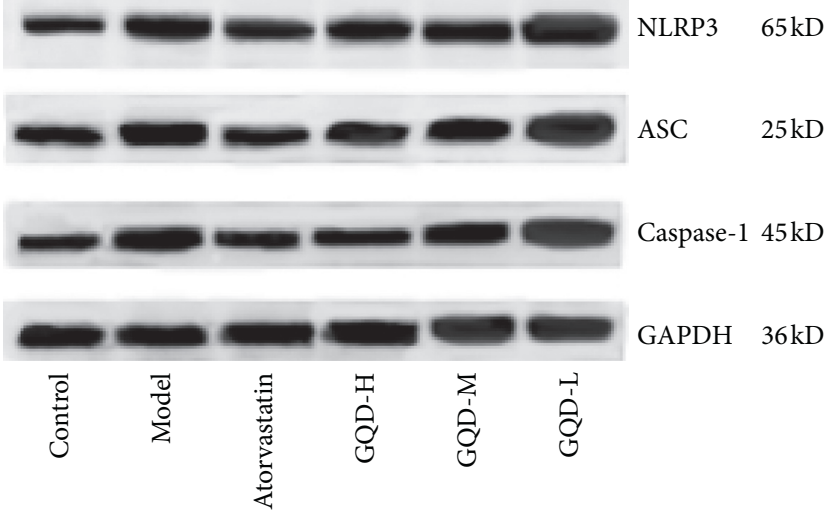

(b)

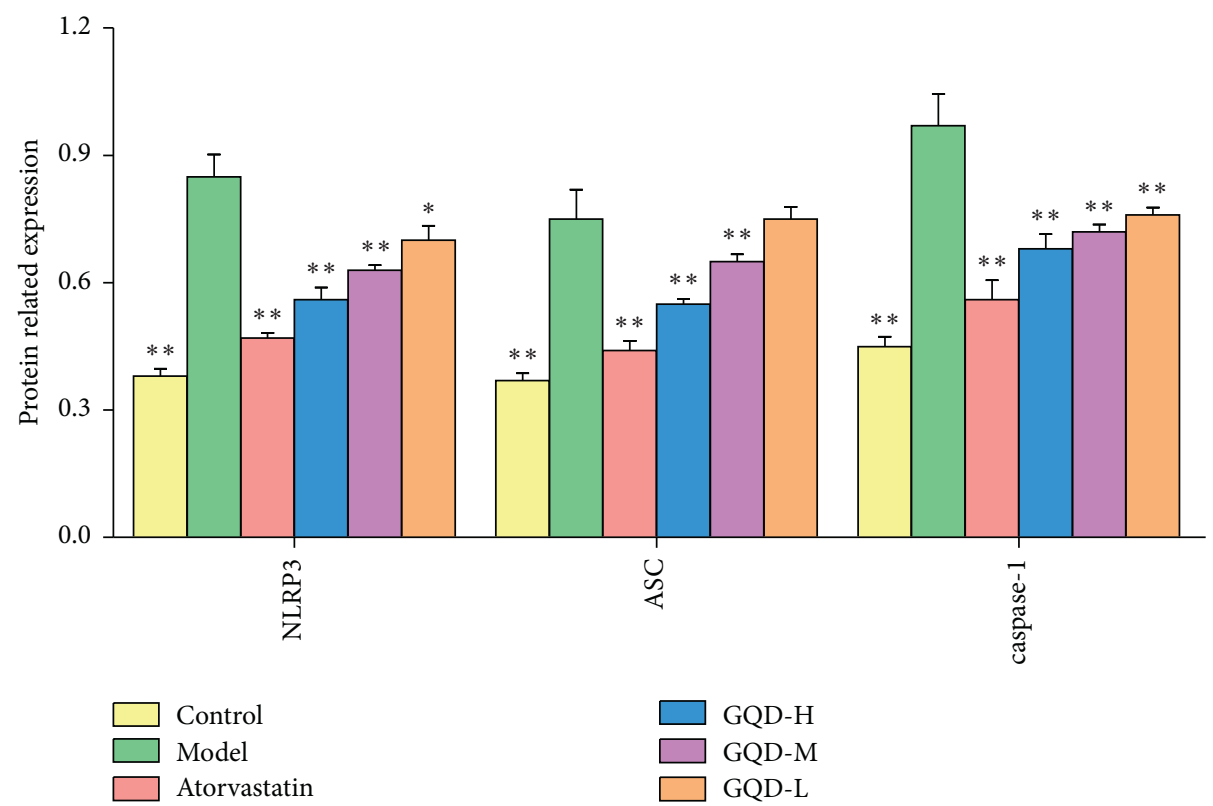

(c)

FIGURE 4: GQD inhibited the NLRP3 signal axis key targets in NAFLD rat liver tissue. (a) Effect of GQD on the NLRP3 signal axis mRNA in liver tissue of NAFLD rat. (b) Western blot graph. (c) Effect of GQD on the NLRP3 signal axis proteins in liver tissue of NAFLD rat. Note: compared with the model group, ${ }^{*} P<0.05 ;{ }^{* *} P<0.01$. 
liver damage [39]. In this regard, the levels of related blood lipid TG, LDL-C, TC, and HDL-C and liver injury indicators ALT, AST, and ALP in rats were investigated. The results found that the levels of TG, LDL-C, TC, ALT, AST, and ALP were significantly reduced, while $\mathrm{HDL}-\mathrm{C}$ was increased after GQD treatment. It can be seen that GQD can mediate the normal metabolism of blood lipids and decrease the liver injury to inhibit NAFLD lesions and prevent their development.

Nucleotide binding oligomerization domain-like receptor protein 3 (NLRP3) is a class of cytoplasmic pattern recognition receptors [40]. Yang et al. reported that NLRP3 signal axis exerted a vital role on NAFLD in a mouse model induced by high-fat diet [40]. Thereinto, caspase-1 is an active product of NLRP3 signal axis, with its adapter protein ASC and procaspase-1, which consist of NLRP3. Studies have shown that NLRP3 knockout in nonalcoholic steatohepatitis (NASH) mice model inhibited the activation of NLRP3, ASC, and caspase-1 in liver and can significantly reduce liver inflammation in mice and prevent the development of the NASH disease [40,41]. Besides, reports have shown that the NLRP3 signal axis is involved in the regulation of important inflammatory cytokines such as IL-6 and IFN- $\beta$ [42-44] and antioxidant factors such as MDA, SOD, and GSH-Px $[25,45]$. Animals fed with HFD has been reported to induce obesity, hyper/dyslipidemia, hepatic steatosis, oxidative stress, mild fibrosis, and enhanced level of cytokines. Thus, amelioration of oxidative stress and inflammation cytokines could be regarded as an antiNAFLD viable mechanism. Collectively, as this regard, blocking the NLRP3 signal axis to antioxidant and antiinflammation is a strategy in treating NAFLD rat model induced by a high-fat diet. Due to the accumulation of triglycerides and free fatty acids in NAFLD rat, excessive reactive oxygen species (ROS) are generated in the liver. The free radical metabolism balance in the body, including SOD and GSH-Px, is mainly maintained by the antioxidant system [46]. MDA is the main product of lipid peroxide degradation, which not only prevents mitochondrial respiratory chain electron transfer but also promotes inflammatory response, thus promoting liver antioxidant enzyme activity (SOD, CAT, and GSH-Px) and inhibiting peroxidation products MDA content and antioxidative stress can promote lipid metabolism and prevent NAFLD caused by obesity $[25,47]$.

Based on those, while studying the regulation of GQD on inflammation and oxidative stress, we further investigated the expression of the NLRP3 signal axis to explore the mechanism of GQD improving NAFLD in rats. The results show that GQD can significantly decrease the serum levels of IFN- $\beta$, TNF- $\alpha$, and MDA in NAFLD rats and increase the expressions of IL-2, SOD, and GSH-Px. GQD can significantly inhibit the expression levels of NLRP3, ASC, and caspase- 1 genes and proteins in liver tissue and block the activation of NLRP3 signal axis. It is suggested that GQD can regulate inflammatory cytokines, inhibit the level of oxidative stress, and downregulate the expressions of key genes and proteins of the NLRP3 signal axis. It has been proved that the above possible anti-NAFLD mechanism strategy is feasible. However, as a multicomponent and multitarget drug, GQD is specific to which component or multiple components playing the effect on NAFLD, and the relevance of the mechanism needs to be deepened.

In conclusion, this study examines the mechanism of GQD treatment of NAFLD from the inflammatory cytokines, oxidative stress, and NLRP3 signal axis. From the animal, molecular, and protein levels, it is initially confirmed that GQD can inhibit NLRP3 signal axis by regulating inflammatory cytokines and oxidative stress. The study well established that GQD improves NAFLD lesions and provides a reference for the research and clinical application of GQD in the treatment of NAFLD. The findings provided scientific evidence in support of GQD being used as one kind of folk remedy to treat NAFLD in China. Further, we will explore how GQD treats NAFLD in connection to the NLRP3 signal axis with inflammation and oxidative stress.

\section{Data Availability}

The datasets used and/or analyzed during the current study are available from the corresponding author upon reasonable request.

\section{Conflicts of Interest}

All authors declare that there are no conflicts of interest regarding the publication of this article.

\section{Authors' Contributions}

Yuqi Ying prepared and wrote the manuscript. Yuqi Ying, Haitao Zhang, Dian Yu, and Wei Zhang contributed to language modification and editing. Yuqi Ying and Shuangchun Liu contributed to study design. Haitao Zhang contributed to the experiment. Yuqi Ying and Dian Yu carried out statistical data analysis. Shuangchun Liu and Dongling Zhou guarantee the integrity of the entire study.

\section{Acknowledgments}

The study was funded by Scientific Research Fund of Taizhou Science and Technology Bureau of Zhejiang Province (no. 1902ky45), Traditional Chinese Medicine Scientific Research Fund of Zhejiang (Class B) (no. 2021ZB332), and Xinmiao Talents Program of Zhejiang Province, China (no. 2019R410013).

\section{Supplementary Materials}

Supplementary Figure S1: the original western blots of the NLRP3 signal axis proteins in liver tissue of NAFLD rat. Supplementary Figure S2: graphical abstract. (Supplementary Materials)

\section{References}

[1] Z. M. Younossi, G. Marchesini, H. Pinto-Cortez et al., "Epidemiology of nonalcoholic fatty liver disease and 
nonalcoholic steatohepatitis: implications for liver transplantation," Transplantation, vol. 103, no. 1, pp. 22-27, 2018.

[2] Y. Dang, J. Xu, M. Zhu, W. Zhou, L. Zhang, and G. Ji, "GanJiang-Ling-Zhu decoction alleviates hepatic steatosis in rats by the miR-138-5p/CPT1B axis," Biomedicine \& Pharmacotherapy, vol. 127, p. 110127, 2020.

[3] Z. Younossi, Q. M. Anstee, M. Marietti et al., "Global burden of NAFLD and NASH: trends, predictions, risk factors and prevention," Nature Reviews Gastroenterology \& Hepatology, vol. 15, no. 1, pp. 11-20, 2018.

[4] H. Hardy, S. Weiskirchen, and R. Weiskirchen, "Current status in testing for nonalcoholic fatty liver disease (NAFLD) and nonalcoholic steatohepatitis (NASH)," Cells, vol. 8, no. 8, p. 845, 2019.

[5] W. Tomeno, K. Kawashima, M. Yoneda et al., "Non-alcoholic fatty liver disease comorbid with major depressive disorder: the pathological features and poor therapeutic efficacy," Journal of Gastroenterology and Hepatology, vol. 30, no. 6, pp. 1009-1014, 2015.

[6] K. Imprialos, K. Stavropoulos, S. Bouloukou, G. Kerpiniotis, A. Karagiannis, and M. Doumas, "Current and potential future pharmacological approaches for non- alcoholic fatty liver disease," Current Vascular Pharmacology, vol. 16, no. 3, pp. 276-288, 2018.

[7] Y. Dang, S. Hao, W. Zhou, L. Zhang, and G. Ji, "The traditional Chinese formulae Ling-gui-zhu-gan decoction alleviated non-alcoholic fatty liver disease via inhibiting PPP1R3C mediated molecules," BMC Complementary and Alternative Medicine, vol. 19, no. 1, p. 8, 2019.

[8] Q. Feng, W. Liu, S. S. Baker et al., "Multi-targeting therapeutic mechanisms of the Chinese herbal medicine QHD in the treatment of non-alcoholic fatty liver disease," Oncotarget, vol. 8, no. 17, pp. 27820-27838, 2017.

[9] Y. Li, W. Guo, C. Zhang et al., "Herbal medicine in the treatment of non-alcoholic fatty liver diseases-efficacy, action mechanism, and clinical application," Frontiers in Pharmacology, vol. 11, p. 601, 2020.

[10] X. Luo, K. Li, B. Zhang, and C. Tang, "[Influencing factors of the occurrence of propagated sensation effects at Zusanli (ST 36) based on artificial intelligence technology]," Chinese Acupuncture and Moxibustion, vol. 38, no. 10, pp. 1105-1108, 2018, (in Chinese).

[11] T. T. Shi, L. Wu, W. J. Ma et al., "Nonalcoholic fatty liver disease: pathogenesis and treatment in traditional Chinese medicine and western medicine," Evidence-Based Complementary and Alternative Medicine, vol. 2020, no. 6, Article ID 8749564, 2020.

[12] Z. Ding, R. Zhong, Y. Yang et al., "Systems pharmacology reveals the mechanism of activity of Ge-Gen-Qin-Lian decoction against LPS-induced acute lung injury: a novel strategy for exploring active components and effective mechanism of TCM formulae," Pharmacological Research, vol. 156, p. 104759, 2020.

[13] Xia, Pharmacopoeia of People's Republic of China, Part 1, pp. 1712-1713, Chemical Industry Press, Beijing, China, 2020.

[14] C. H. Zhang, G. L. Xu, Y. H. Liu et al., "Antidiabetic activities of Gegen Qinlian Decoction in high-fat diet combined with streptozotocin-induced diabetic rats and in 3T3-L1 adipocytes," Phytomedine, vol. 20, no. 3-4, pp. 221-229, 2013.

[15] T. Liu, X. Tian, Z. Li et al., "Metabolic profiling of Gegenqinlian decoction in rat plasma, urine, bile and feces after oral administration by ultra high performance liquid chromatography coupled with Fourier transform ion cyclotron resonance mass spectrometry," Journal of Chromatography B, vol. 1079, pp. 69-84, 2018.

[16] C. S. Liu, X. Liang, X. H. Wei et al., "Gegen Qinlian decoction treats diarrhea in piglets by modulating gut microbiota and short-chain fatty acids," Frontiers in Microbiology, vol. 10, p. 825, 2019.

[17] B.-L. Xu, G.-J. Zhang, and Y.-B. Ji, "Active components alignment of Gegenqinlian decoction protects ulcerative colitis by attenuating inflammatory and oxidative stress," Journal of Ethnopharmacology, vol. 162, pp. 253-260, 2015.

[18] J. A. Ryuk, M. Lixia, S. Cao, B.-S. Ko, and S. Park, "Efficacy and safety of Gegen Qinlian decoction for normalizing hyperglycemia in diabetic patients: a systematic review and meta-analysis of randomized clinical trials," Complementary Therapies in Medicine, vol. 33, pp. 6-13, 2017.

[19] X. Ko, W. Yang, H. Liu et al., "Antilipemic effect of gengenqinlian decoction in hyperlipidemia rats," Asia-Pacific Traditional Medicine, vol. 8, no. 11, pp. 12-14, 2012, (in Chinese).

[20] C.-H. Zhang, Q. Xiao, J.-Q. Sheng et al., “Gegen Qinlian Decoction abates nonalcoholic steatohepatitis associated liver injuries via anti-oxidative stress and anti-inflammatory response involved inhibition of toll-like receptor 4 signaling pathways," Biomedicine \& Pharmacotherapy, vol. 126, p. 110076, 2020.

[21] Y. Liu, P. H. Ding, L. J. Liu et al., "Gegen qinlian decoction attenuates high-fat diet-induced steatohepatitis in rats via gut microbiota," Evidence-based Complementary and Alternative Medicine, vol. 2018, no. 3, 8 pages, Article ID 7370891, 2018.

[22] S. Wang, Y. K. Lin, X. Yuan, F. Li, L. Guo, and B. Wu, "REV$\mathrm{ERB} \alpha$ integrates colon clock with experimental colitis through regulation of NF- $\kappa \mathrm{B} / \mathrm{NLRP} 3$ axis," Nature Communications, vol. 9, no. 1, p. 4246, 2018.

[23] T. Zhang, H. Du, S. Feng et al., "NLRP3/ASC/Caspase-1 axis and serine protease activity are involved in neutrophil IL- $1 \beta$ processing during Streptococcus pneumoniae infection," Biochemical and Biophysical Research Communications, vol. 513, no. 3, pp. 675-680, 2019.

[24] J. Peng, "The pharmacological targets and clinical evidence of natural products with anti-hepatic inflammatory properties," Frontiers in Pharmacology, vol. 9, p. 455, 2018.

[25] D. K. Dwivedi and G. B. Jena, "NLRP3 inhibitor glibenclamide attenuates high-fat diet and streptozotocin-induced non-alcoholic fatty liver disease in rat: studies on oxidative stress, inflammation, DNA damage and insulin signalling pathway," Naunyn-Schmiedeberg's Archives of Pharmacology, vol. 393, no. 4, pp. 705-716, 2020.

[26] Y. Yan, C. Du, Z. Li et al., "Comparing the antidiabetic effects and chemical profiles of raw and fermented Chinese Ge-GenQin-Lian decoction by integrating untargeted metabolomics and targeted analysis," Chinese Medicine, vol. 13, no. 1, p. 54, 2018.

[27] M. Efati, M. Khorrami, A. Z. Mahmmudabadi, and J. R. Sarshoori, "Induction of an animal model of non-alcoholic fatty liver disease using a formulated high-fat diet," Journal of Babol University of Medical Sciences, vol. 18, no. 11, pp. 57-62, 2016.

[28] L. Li, D. Z. Lu, Y. M. Li, X.-Q. Zhang, X.-X. Zhou, and X. Jin, "Proteomic analysis of liver mitochondria from rats with nonalcoholic steatohepatitis," World Journal of Gastroenterology, vol. 20, no. 16, pp. 4778-4786, 2014.

[29] M. W. Pfaffl, "A new mathematical model for relative quantification in real-time RT-PCR," Nucleic Acids Research, vol. 29, no. 9, p. e45, 2001. 
[30] P. Angulo, "Nonalcoholic fatty liver disease," New England Journal of Medicine, vol. 346, no. 16, pp. 1221-1231, 2002.

[31] W. Huang and X. X. Zhang, "Research advances in the pathogenesis of non-alcoholic fatty liver disease-associated liver cancer," Chinese Journal Hepatology, vol. 25, no. 2, pp. 157-160, 2017.

[32] Y. Sumida and M. Yoneda, "Current and future pharmacological therapies for NAFLD/NASH," Journal of Gastroenterology, vol. 53, no. 3, pp. 362-376, 2018.

[33] S. L. Friedman, B. A. Neuschwander-Tetri, M. Rinella, and A. J. Sanyal, "Mechanisms of NAFLD development and therapeutic strategies," Nature Medicine, vol. 24, no. 7, pp. 908-922, 2018.

[34] J.-M. Yang, Y. Sun, M. Wang et al., "Regulatory effect of a Chinese herbal medicine formula on non-alcoholic fatty liver disease," World Journal of Gastroenterology, vol. 25, no. 34, pp. 5105-5119, 2019.

[35] J. E. Lambert, M. A. Ramos-Roman, J. D. Browning, and E. J. Parks, "Increased De novo lipogenesis is a distinct characteristic of individuals with nonalcoholic fatty liver disease," Gastroenterology, vol. 146, no. 3, pp. 726-735, 2014.

[36] A. Rahimi, M. M. Shabestari, K. Faryadian, V. Safaeinejad, J. S. Moazen, and Z. Fallah, "The effect of selecting aerobics exercise program (walking in water and in land) on HDL-C, LDL-C, TC and TG in non-athlete menopausal women," European Journal of Experimental Biology, vol. 3, no. 2, pp. 463-468, 2013.

[37] H. M. Yan, M. F. Xia, Y. Wang et al., "Efficacy of Berberine in patients with non-alcoholic fatty liver disease," PLoS One, vol. 10, no. 8, p. e0134172, 2015.

[38] N. Reddy, P. Naylor, Z. Hakim et al., "Effect of Treatment for $\mathrm{CHC}$ on liver disease progression and hepatocellular carcinoma development in african americans," Journal of Clinical and Translational Hepatology, vol. 3, no. 3, pp. 163-168, 2015.

[39] S. Wang, J. Zhang, L. Zhu et al., "Association between liver function and metabolic syndrome in Chinese men and women," Scientific Reports, vol. 7, p. 44844, 2017.

[40] G. Yang, H. E. Lee, and J. Y. Lee, "A pharmacological inhibitor of NLRP3 inflammasome prevents non-alcoholic fatty liver disease in a mouse model induced by high fat diet," Scientific Reports, vol. 6, p. 24399, 2016.

[41] T. Deng, J. P. Gong, and K. He, "Effect of knockout of NLRP3 gene on non-alcoholic steatohepatitis mice," Shandong Medicine Journal, vol. 59, no. 1, pp. 1-4, 2019, (in Chinese).

[42] K. Álvarez and G. Vasquez, "Damage-associated molecular patterns and their role as initiators of inflammatory and autoimmune signals in systemic lupus erythematosus," International Reviews of Immunology, vol. 36, no. 5, pp. 259-270, 2017.

[43] C. J. Zuurbier, W. M. C. Jong, O. Eerbeek et al., "Deletion of the innate immune NLRP3 receptor abolishes cardiac ischemic preconditioning and is associated with decreased Il-6/ STAT3 signaling," PLoS One, vol. 7, no. 7, p. e40643, 2012.

[44] N.-H. Oh, J.-W. Han, D.-W. Shim et al., "Anti-inflammatory properties of Morus bombycis Koidzumi via inhibiting IFN- $\beta$ signaling and NLRP3 inflammasome activation," Journal of Ethnopharmacology, vol. 176, pp. 424-428, 2015.

[45] S. Sim, J. Wang, C. Hao, H. Dang, and S. Jiang, “Tetramethylpyrazine ameliorates depression by inhibiting TLR4NLRP3 inflammasome signal pathway in mice," Psychopharmacology, vol. 236, no. 7, pp. 2173-2185, 2019.

[46] G. Dang, L. G. D. Pasqua, C. Berardo et al., "Animal models of steatosis (NAFLD) and steatohepatitis (NASH) exhibit hepatic lobe-specific gelatinases activity and oxidative stress,"
Canadian Journal of Gastroenterology and Hepatology, vol. 3, p. 5413461, 2019.

[47] J.-M. Yin, L.-B. Sun, J.-S. Zheng, X.-X. Wang, D.-X. Chen, and N. Li, "Copper chelation by trientine dihydrochloride inhibits liver RFA-induced inflammatory responses in vivo," Inflammation Research, vol. 65, no. 12, pp. 1009-1020, 2016. 